\title{
GENERATION RECOMBINATION NOISE IN GaN PHOTOCONDUCTING DETECTORS
}

\author{
M. Misra, D. Doppalapudi, A.V. Sampath, T.D. Moustakas \\ Department of Electrical Engineering and Center for Photonics Research, Boston University, MA \\ 02215 \\ P.H. McDonald \\ Center for Photonics Research, Boston University, MA 02215
}

Cite this article as: MRS Internet J. Nitride Semicond. Res. 4S1, G7.8 (1999)

\begin{abstract}
$\underline{\text { Abstract }}$
Low frequency noise measurements are a powerful tool for detecting deep traps in semiconductor devices and investigating trapping-recombination mechanisms. We have performed low frequency noise measurements on a number of photoconducting detectors fabricated on autodoped $\mathrm{n}-\mathrm{GaN}$ films grown by ECR-MBE. At room temperature, the noise spectrum is dominated by $1 / \mathrm{f}$ noise and thermal noise for low resistivity material and by generationrecombination (G-R) noise for high resistivity material. Noise characteristics were measured as a function of temperature in the $80 \mathrm{~K}$ to $300 \mathrm{~K}$ range. At temperatures below $150 \mathrm{~K}, 1 / \mathrm{f}$ noise is dominant and at temperatures above $150 \mathrm{~K}$, G-R noise is dominant. Optical excitation revealed the presence of traps not observed in the dark, at room temperature.
\end{abstract}

\section{Introduction}

The III-Nitrides is an important class of materials for the development of optoelectronic devices and electronic devices for operation at high speeds and high temperatures ${ }^{1}$. The performance of most of these devices is strongly influenced by the presence of defects and traps in the material. Several techniques, such as Deep Level Transient Spectroscopy (DLTS) ${ }^{2,-4}$, Photo Induced Capacitance Transient Spectroscopy (PICTS) ${ }^{5}$, Thermally Stimulated Current (TSC) ${ }^{6}$, and Thermal Admittance Spectroscopy (TAS) ${ }^{7}$ have been used to investigate and characterize the deep defects in GaN. In this paper, we describe the technique of noise spectroscopy to study the presence of deep levels in GaN films grown on sapphire. Noise spectroscopy has been used as a tool to investigate traps in silicon ${ }^{8}$ and III-As/P compounds, and to study the influence of the deep levels on performance of devices such as JFETs ${ }^{9}$ and MODFETs ${ }^{10,11}$. It has also been used to characterize the performance of GaN p-n junctions. ${ }^{15}$ To the best of our knowledge, this is the first report of using noise measurements to study deep levels in $\mathrm{GaN}$ photoconductors.

\section{Theory}

Photoconducting detectors, typically, exhibit three types of noise: $1 / f$ noise which is associated with contacts and surfaces, generation-recombination (G-R) noise which is associated with the trapping and emission of charges from deep traps and thermal or Johnson noise, which is associated with the resistance of the material. Generation-recombination noise arises because the trapping and emission of charges from the deep levels causes fluctuations in the dc dark current. This excess current decays, on an average, with a time constant $\tau$, so that the average current as a function of time $t$ will be given as

$$
i(t)=I_{0} \exp (-t / \tau)
$$


The time constant $\tau$ is a characteristic of the deep level and is related to its emission $\left(\tau_{\mathrm{e}}\right)$ and capture $\left(\tau_{\mathrm{c}}\right)$ time constants according to the relation ${ }^{12}$

$$
\frac{1}{\tau}=\frac{1}{\tau e}+\frac{1}{\tau c}
$$

When the noise arises from deep levels with a well-defined time constant the observed noise spectrum has a Lorentzian shape which can be described as

$$
\left\langle i^{2}\right\rangle=\frac{A * \Delta f}{\left[1+\left(f / f_{0}\right)^{2}\right]}
$$

where $f_{0}=1 / 2 \pi \tau$ is the characteristic frequency associated with the time constant $\tau$ and $A$ is the amplitude of the noise current at frequencies $\mathrm{f}<<\mathrm{f}_{0}$, and referred to as the zero frequency noise plataeu. It is from the variation of the zero frequency plataeu value A and the time constant $\tau$ with temperature that the activation energy of deep levels can be determined. If the variation of the free carrier concentration $\mathrm{n}$ as a function of temperature is also known, other trap parameters such as its density, degeneracy and capture cross section can also be deduced. Furthermore, multi-level spectra can be decomposed into separate Lorentzians, such that each Lorentzian contains information of a single trap, if the Fermi level is a few kT away from the trap level and the concentration of mobile carriers is larger than the concentration of impurities ${ }^{13}$.

The zero frequency amplitude increases as the temperature increases until the Fermi-level crosses the trap level, and then decreases rapidly. ${ }^{12,13}$ The temperature dependence of $\tau$ is obtained from the following relation ${ }^{13}$,

$$
\frac{1}{\tau}=\alpha n\left[B \exp \left(\frac{E_{T}-E_{f}}{k T}\right)+1\right]
$$

where $\alpha$ is the recombination coefficient, $\mathrm{B}$ and $\mathrm{E}_{\mathrm{T}}$ are the degeneracy factor and depth of the trap respectively, $\mathrm{E}_{\mathrm{f}}$ is the dark Fermi-level, $\mathrm{n}$ is the free carrier concentration, $\mathrm{k}$ is Boltzman's constant, $\mathrm{T}$ is the temperature. In the low temperature limit, when the Fermi-level lies above the trap level, the exponential in Eq. (4) is small compared to unity and the characteristic time constant is virtually constant. As the temperature increases, and the Fermi-level is below the trap level, the exponential dominates, and a strong temperature dependence is observed.

In order to extract trap parameters, we followed the method derived by Copeland ${ }^{12}$. In brief, the method consists of combining the three temperature dependent experimentally observed parameters $A, \tau$ and $n$ into a single function of temperature $F(T)$, such that

$$
F(T)=\frac{A n^{2}}{\tau}
$$

From the slope of the plot of $\ln (\mathrm{F}(\mathrm{T}))$ versus $1 / \mathrm{T}$, the trap activation energy and other parameters can be determined.

\section{$\underline{\text { Experimental Methods }}$}

Noise measurements were performed on photoconducting detectors fabricated on $\mathrm{n}-\mathrm{GaN}$ films grown on c-plane sapphire by the electron cyclotron resonance microwave plasma assisted molecular beam epitaxy (ECR-MBE) method. The photoconducting detectors were fabricated by patterning interdigitated electrodes, with inter-electrode spacing ranging from $5 \mu \mathrm{m}$ to $20 \mu \mathrm{m}$, 
using standard photolithography and lift-off techniques. For the noise measurements, we studied two types of devices: (i) devices fabricated on low resistivity material $\left(\rho=10^{2} \mathrm{ohm}-\mathrm{cm}\right.$ ) and (ii) devices fabricated on high resistivity material $\left(\rho=10^{6} \mathrm{ohm}-\mathrm{cm}\right)$. The noise characteristics of the $\mathrm{GaN}$ photoconducting detectors were measured in the $10 \mathrm{~Hz}$ to $50 \mathrm{KHz}$ range using a low noise current preamplifier and a signal analyzer. The device was mounted in a temperature controlled cryostat and the temperature was varied from $80 \mathrm{~K}$ to $320 \mathrm{~K}$. The bias voltage on the sample varied from $0.5 \mathrm{~V}$ to $1.5 \mathrm{~V}$. In order to illuminate the photoconductors, a $10 \mathrm{~mW} \mathrm{He}-\mathrm{Cd}$ laser, emitting at $325 \mathrm{~nm}$ was used.

\section{$\underline{\text { Experimental Results }}$}

In this section, we shall illustrate (i) the effect of the position of the dark Fermi-level, (ii) the effect of optical excitation and (iii) the effect of thermal excitation on the noise characteristics of GaN photoconducting detectors.
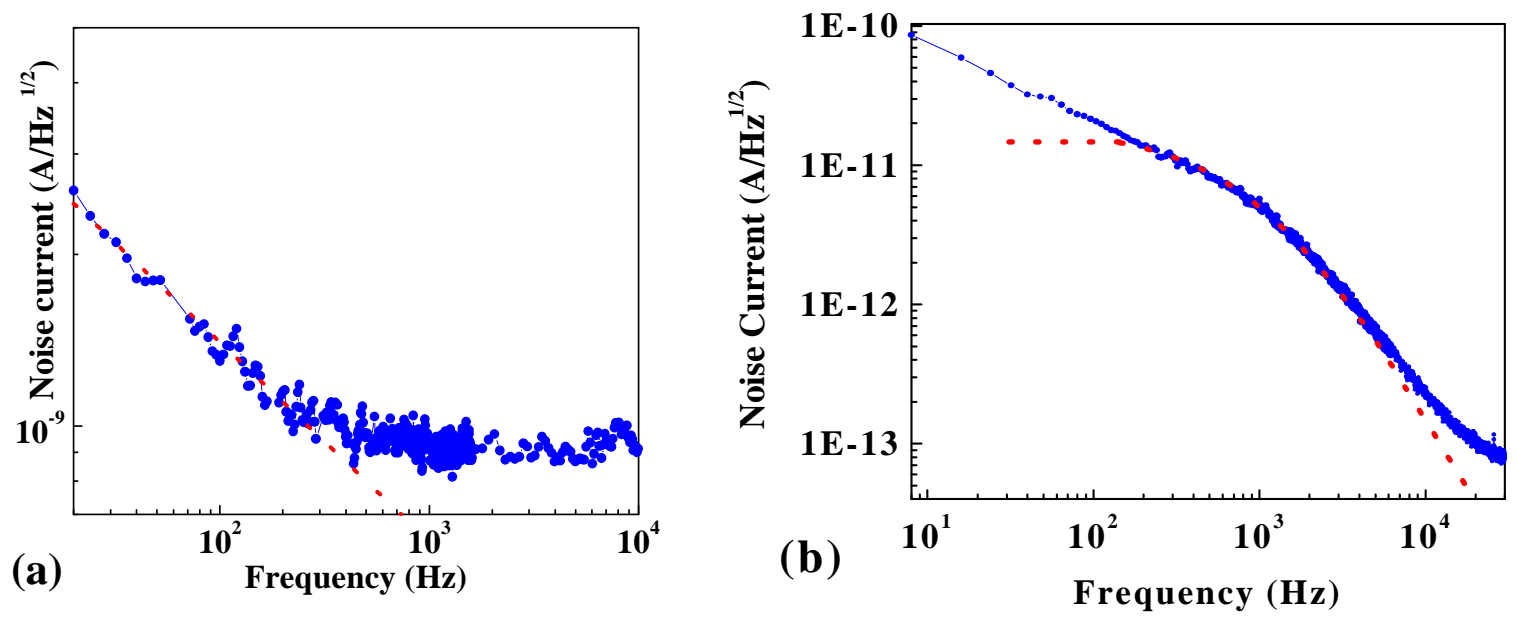

Figure 1: Noise characteristics at room temperature for $\mathrm{GaN}$ photoconducing detectors fabricated on (a) low resistivity material and (b) on high resistivity material. Dotted lines are (a) linear and (b) lorentzian fit to the data.

Figure 1a shows the noise spectrum at room temperature for a $\mathrm{GaN}$ photoconducting detector fabricated on low resistivity material. The spectrum is dominated by $1 / \mathrm{f}$ noise in the low frequency range $(f<500 \mathrm{~Hz})$ and by thermal noise at higher frequencies $(\mathrm{f}>500 \mathrm{~Hz})$. The dark Fermi-level, at room temperature, was estimated to be $0.18 \mathrm{eV}$ below the conduction band. Since the noise measurement is sensitive to levels within a few kT of the Fermi-level, this measurement senses shallow traps, which typically have higher characteristic frequencies. Since the thermal noise is quite large, the G-R noise from these shallow levels is obscured at the higher frequencies. Figure $1 \mathrm{~b}$ shows the noise spectrum for a $\mathrm{GaN}$ photoconducting detector fabricated on high resistivity material, measured under identical conditions. In this case, the spectrum shows $1 / \mathrm{f}$ noise in the $10 \mathrm{~Hz}-100 \mathrm{~Hz}$ range and a strong contribution of G-R noise in the $100 \mathrm{~Hz}-$ $10 \mathrm{KHz}$ range. In this case the dark Fermi-level was estimated to be $0.38 \mathrm{eV}$ below the conduction band edge. Thus it measures G-R noise from the deep levels, within a few kT of the Fermi-level. In addition, the thermal noise level, evident at frequencies greater than $20 \mathrm{KHz}$ is much lower. By fitting a Lorentzian to the measured spectrum (dotted line), we deduced a characteristic frequency of $1.5 \mathrm{KHz}$ for this trap, corresponding to a characterisitic time constant of $106 \mu \mathrm{s}$. 
The effect of illumination is to excite traps throughout the gap and the traps for which the G-R noise contribution is greater than the thermal noise, will be observed. Figure 2 illustrates the noise characteristics from optically excited traps for both samples discussed earlier. We find that the low resistivity sample shows a G-R noise component with a characteristic frequency of about $1.5 \mathrm{KHz}$ upon optical excitation. The high resistivity device shows two G-R noise components: (i) with a characteristic frequency at $1.5 \mathrm{KHz}$ as seen by thermal excitation and an additional shallower level with a characteristic frequency of about $10 \mathrm{KHz}$, which was not observed by thermal excitation alone at room temperature.

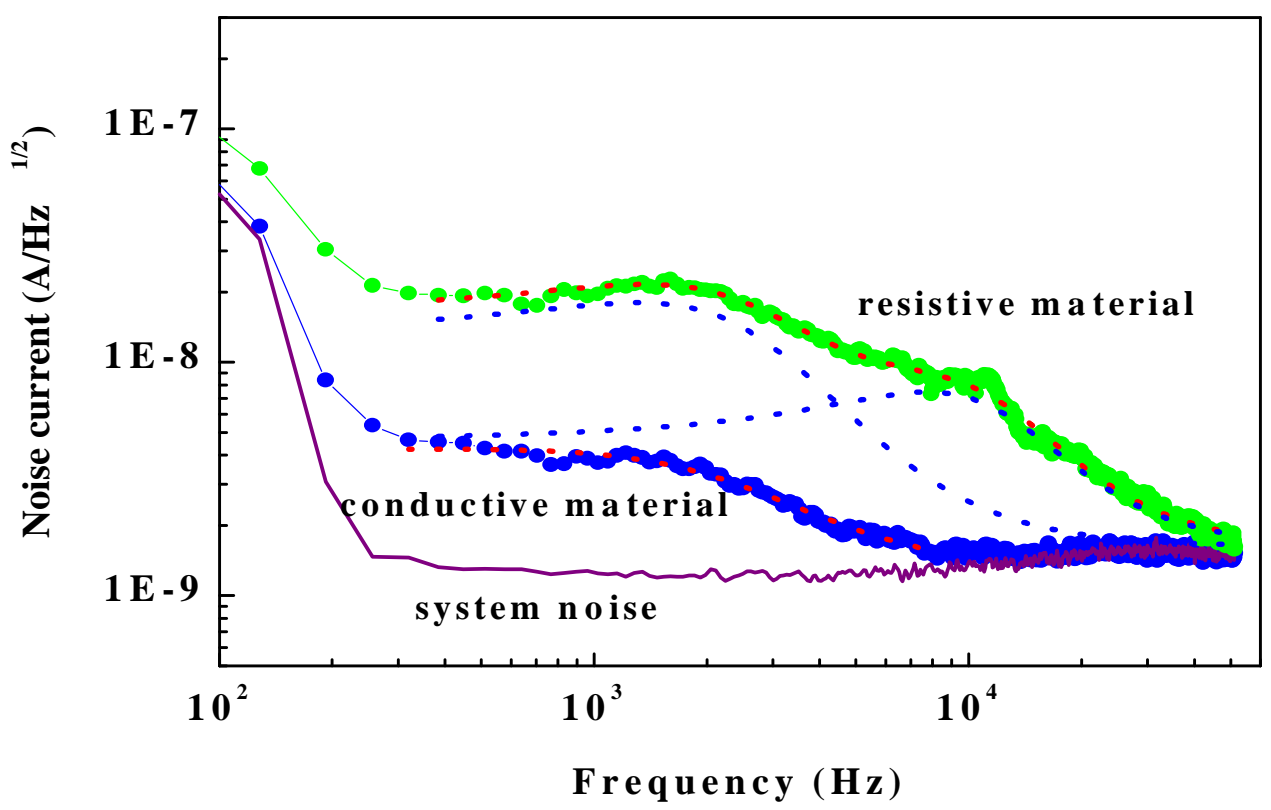

Figure 2: Noise characteristics of the GaN photoconductors when illuminated with He-Cd laser.

From the similarity in the characteristic frequency for the two samples, we may infer that the same trap level is present in both detectors and responsible for the G-R noise observed.

The temperature dependence of the noise characteristics of the high resistivity detector in the $80 \mathrm{~K}$ to $300 \mathrm{~K}$ range was also investigated. At temperatures below $120 \mathrm{~K}$, the noise spectrum was dominated by $1 / \mathrm{f}$ noise and thermal noise, and no G-R noise component was observable. At temperatures higher than $120 \mathrm{~K}$, a gradual increase in the G-R noise component was increased. An increase in the zero-frequency plataeu value and and shift in the characteristic frequency was observed. Figures $3 \mathrm{a}$ and $3 \mathrm{~b}$ show the noise characteristics at $100 \mathrm{~K}$ and $160 \mathrm{~K}$. The same at $300 \mathrm{~K}$ are shown in figure $1 \mathrm{~b}$. In addition, we also measured the variation in the dark resistance of the detector as a function of temperature in the $80 \mathrm{~K}-300 \mathrm{~K}$ range, to obtain an independent measurement of the free electron concentration $n$. From the variation of $A, f_{0}$ and $n$, we calculated the function $\mathrm{F}(\mathrm{T})$ and estimate the depth of the trap to be $0.38 \mathrm{eV}$. In addition, from the measurement of the characteristic frequency and the measurement of the free carrier concentration at room temperature, we can calculate the depth of the trap using Eq. (4).

Using this expression, with $\alpha=3 \times 10^{-11} \mathrm{~cm}^{3} / \mathrm{s},{ }^{14} \mathrm{n}=1.5 \times 10^{15} \mathrm{~cm}^{-3}, B=1, \mathrm{~T}=300 \mathrm{~K}$, we calculated the depth of the trap to be $0.4 \mathrm{eV}$ below the conduction band. This value is in good agreement with the observation of a deep level in the $0.4 \mathrm{eV}-0.5 \mathrm{eV}$ range by several researchers 


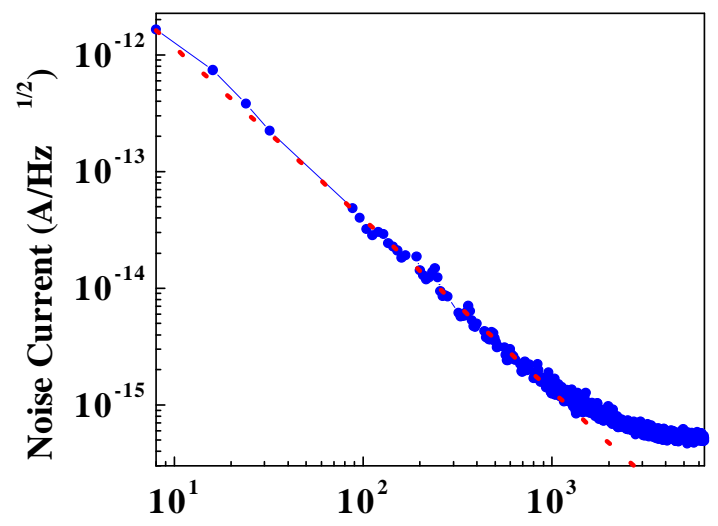

(a)

Frequency $(\mathbf{H z})$

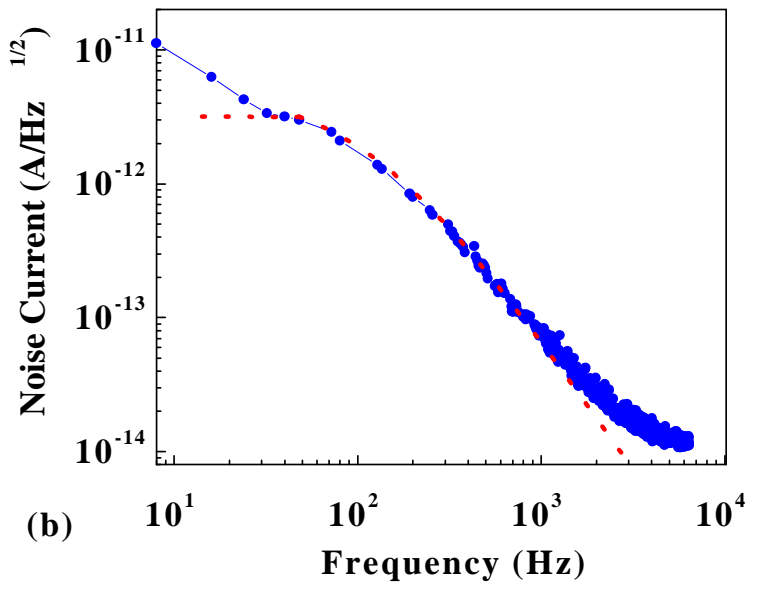

Figure 3: Noise characteristics of the resistive device at (a) $100 \mathrm{~K}$ and (b) $160 \mathrm{~K}$. Dotted lines are (a) linear and (b) Lorentzian fits to the data.

using other techniques such as DLTS, PICTS and TAS, on samples grown by MOVPE ${ }^{2-5}$ and MBE. ${ }^{6}$ and in GaN p-n junctions operating in the forward bias, grown by MOCVD, and studied used techniques similar to those described in this paper. ${ }^{15}$

From the relation $\tau=1 / \mathrm{v}_{\mathrm{th}} \mathrm{Sn}$, where $\mathrm{v}_{\mathrm{th}}$ is the thermal velocity of electrons, we have calculated the capture cross-section to be $S=2 \times 10^{-19} \mathrm{~cm}^{2}$, which indicates that the trap is a coulombic center. However, we believe that measurements above $300 \mathrm{~K}$ are necessary to observe the sharp decrease in $\tau$ and A with temperature, as the Fermi-level crosses the trap level. Unfortunately, we could not measure the noise characteristics at higher temperatures, at this time, due to excessive $1 / \mathrm{f}$ noise in our high temperature apparatus

\section{Conclusions}

In conclusion, we have investigated the noise behavior of $\mathrm{GaN}$ photoconductors in the $10 \mathrm{~Hz}-50 \mathrm{KHz}$ range and was found that it is dominated by $1 / f$ and thermal noise for low resistivity $\left(\rho=10^{2}\right.$ ohm-cm) devices and by G-R noise for high resistivity devices $\left(\rho=10^{6}\right.$ ohm-cm) at room temperature. A deep level was identified, with a time constant of $106 \mu \mathrm{s}$, and its activation energy was estimated to be in the $0.3 \mathrm{eV}-0.4 \mathrm{eV}$ range. The capture cross-section of this trap was found to be $2 \times 10^{-19} \mathrm{~cm}^{2}$, suggesting that it is a coulombic center. In addition, optical excitation revealed the presence of a similar trap in the low resistivity material, which was not seen by thermal excitation. However, noise measurements need to be performed at temperatures higher than $300 \mathrm{~K}$, to determine the full temperature dependence of $\tau$ and to confirm the trap properties. To the best of our knowledge, this is the first report of using noise measurements to study deep levels in GaN photoconductors.

\section{Acknowledgements}

This work was supported by DARPA through a sub-contract with Lockheed-Martin. 


\section{$\underline{\text { References }}$}

1. H. Morkoc, S. Strite, G.B. Gao, M.E. Lin, B. Sverdlov and M. Burns, J. Appl. Phys. 76, 1363, (1994)

2. P. Hacke, H. Nakayama, T. Detchprom, K. Hiramatsu and N. Sawaki, Appl. Phys. Lett. 68, 1362, (1996)

3. W.I. Lee, T.C. Huang, J.D. Guo and M.S. Feng, Appl. Phys. Lett. 67, 1721, (1995)

4. W. Gotz, N.M. Johnson, R.A. Street, H. Amano and I. Akasaki, Appl. Phys. Lett. 66, 1340, (1995)

5. A.Y. Polyakov, N.B. Smirnov, A.V. Govorkov, M. Shin, M. Skowronski and D.W. Greve, J. Appl. Phys. 84, 870, (1998)

6. D.C. Look, Z.-Q. Fang, W. Kim, O. Atkas, A. Botchkarev, A. Salvador and H. Morkoc, Appl. Phys. Lett. 68, 3775, (1996)

7. A. Krtschil, H. Witte, M. Lisker, J. Christen, U. Birkle, S. Einfeldt, D. Hommel, , J. Appl. Phys. 84, 2040, (1998)

8. S.L. Jang, and G. Bosman, J. Appl. Phys. 65, 201, (1989)

9. S. Kugler, K. Steiner, U. Seiler, K. Heime and E. Kuphal, Appl. Phys. Lett. 52, 111, (1988)

10. J.R. Kirtley, T.N. Theis, P.M. Mooney, S.L. Wright, J. Appl. Phys. 63, 1541, (1988)

11. S. Kugler, IEEE Trans. Electron. Dev. 35, 623, (1988)

12. J. A. Copeland, IEEE Trans. Electron. Dev. ED-18, 50, (1971)

13. A.D. vanRheenen, G. Bosman, R.J. Zijlstra, Solid State Electron. 30, 259, (1987)

14. A.V. Dmitriev, A.L. Oruzheinikov, M.V. Lomonosov, MIJ-NSR 1, 46, (1996)

15. D.V. Kuksenkov, H. Temkin, A. Osinsky, R. Gaska, M.A. Khan, J. Appl. Phys. 83, 2142, (1998) 